\title{
El compromiso de la masonería con la educación a finales del siglo XIX. EI caso de la logia América de Ubrique (Cádiz)
}

\section{The commitment of Freemasonry with education at the end of the 20th century. The case of the America lodge of Ubrique (Cádiz)}

\author{
Antonio Morales Benítez \\ Doctor en historia por la Universidad de Cádiz, España, 2016. Correo electrónico: anmobeni@hotmail.com \\ DOI: http://dx.doi.org/10.15517/rehmlac.v9i1.28177
}

Fecha de recibido: 10 de febrero de 2017 - Fecha de aceptación: 23 de marzo de 2017

\section{Palabras clave}

Masonería; educación; círculos culturales; cuestión social; Sierra de Cádiz

\section{Keywords}

Freemasonry; education; cultural circles; social issue; Sierra de Cádiz.

\section{Resumen}

Este trabajo analiza la actividad de la logia América en una localidad de la Andalucía rural tendente a la extensión de la educación a aquellos que no tenían acceso a la cultura, a través de diversas instituciones patrocinadas por la propia masonería, como medio o instrumento para combatir los males que aquejaban a la población formando a ciudadanos que asegurasen su desarrollo.

\begin{abstract}
The present paper analyzes the activity of the America lodge in a locality of rural Andalusia. It provided further education to those who were unable to have access to culture through diverse institutions sponsored by Freemasonry as a means or instrument to fight the misfortunes, which afflicted the population by training and educating citizens to ensure their development.
\end{abstract}

\section{Introducción}

Uno de los principales cometidos de la masonería es la formación de sus miembros y la proyección de las enseñanzas y valores asimilados en el interior de los talleres parece una consecuencia lógica ${ }^{1}$. Un camino que lleva a los masones a influir en el resto de la

\footnotetext{
${ }^{1}$ Antonio Morales Benítez, "Masonería y Educación. La propuesta pedagógica de la logia América de Ubrique a finales del siglo XIX", Hespérides 15 (2013): 22-26.
} 
sociedad $^{2}$. Sin embargo, esta vocación social no siempre parece ser el resultado de un proceso previo. En el caso de la logia América de Ubrique muchos de sus integrantes se aproximaron a esta institución no solo buscando un perfeccionamiento personal, sino que, y de una manera paralela, la mejora de las condiciones materiales de la población. Incluso, parece que lo segundo puede ser prioritario, dada la gravedad y la degradación de las relaciones sociales.

Se buscaba directamente en la orden una vía alternativa al Estado para intervenir en algunas cuestiones básicas que impedían el desarrollo de los pueblos. Se concibe, por tanto, la institución masónica como un instrumento válido para la mejora de la sociedad en su conjunto. Tal sería la atracción que ejerció la orden en esta zona de la Serranía de Cádiz que pudo ser el vehículo utilizado por una pequeña burguesía ilustrada con inquietudes políticas, sociales y culturales. A través de este instrumento muchos llegaron a la conclusión que todas las cuestiones pendientes tenían un punto en común o se reducían a la falta de instrucción de numerosas capas de la sociedad. Por lo que la cuestión educativa será un asunto central para los masones de esta localidad serrana al considerarlo como verdadero motor de transformación social.

\section{Escuela y masonería}

La logia América quiso materializar sus ideales de progreso patrocinando centros educativos, así como otras iniciativas tendentes a intervenir en la vida pública ubriqueña a través de instituciones culturales. Estas entidades presentaban unas características comunes como el laicismo o su redentorismo social. Su objetivo final debía ser el progreso de la población a través de una nueva generación de ciudadanos formados en estas instituciones.

A fines del siglo XIX esta localidad gaditana estaba inmensa en un entorno económico deprimido y casi marginal, en clara regresión demográfica. Al deterioro de la economía habría que añadir también el de las relaciones sociales, puesto que estaba sumida en una realidad dominada por la estructura caciquil con el poder político concentrado en unas pocas familias. Y en el otro extremo del tejido social habría que destacar el arraigo de las organizaciones anarquistas ${ }^{3}$.

En este contexto, la introducción de la masonería supuso un indudable impacto e iba a estar sustentada por una clase media formada por una pequeña burguesía industrial, profesiones liberales y propietarios. Este sector inquieto cultural y políticamente interpretó

\footnotetext{
${ }^{2}$ Sobre este tema véase Pedro Álvarez Lázaro, La Masonería, escuela de formación del ciudadano. La educación interna de los masones españoles en el último tercio del siglo XIX (Madrid: Publicaciones de la Universidad Pontificia Comillas, 1996); José Ignacio Cruz Orozco, Masonería y educación en la II República española (Alicante: Instituto de Cultura Juan Gil Albert, 1993).

${ }^{3}$ Sabemos de la existencia en esta localidad de sociedades adscritas a la AIT desde 1871 y a la FTRE desde 1882.
} 
que sus fines dentro de la masonería no debían ser otros que la regeneración social a través de instituciones educativas y culturales.

La logia ubriqueña estuvo presente en esta localidad de una manera casi ininterrumpida durante 45 años. Dada su larga trayectoria, llegó a ser considerada como la más antigua de toda Andalucía. Durante estos años acogió a más de un centenar de miembros, intervino en numerosos foros y por su activismo llegó a ser una de las más mencionadas en los boletines de las federaciones y consiguientemente gozó de un merecido prestigio en los círculos masónicos de toda España ${ }^{4}$.

América no. 189 de Ubrique se constituyó en 1882 auspiciada por el Gran Oriente de España ${ }^{5}$. Su fundación se debe al propietario cubano Alejo Bonachea Palmero, de nombre simbólico Perseverancia, que es el primer masón residente en Ubrique del que tenemos noticias, y también el único con residencia en la provincia de Cádiz que participó en la fundación de la logia Fiat Lux de Ronda. Bonachea se había iniciado años atrás en la isla de Cuba, perteneciendo a una logia denominada América no. 13 de su localidad natal, San Juan de los Remedios ${ }^{6}$, fundada en 1865 dentro del Gran Oriente de Colón ${ }^{7}$.

A partir de 1888 el taller iniciaría una nueva etapa caracterizada por una cierta renovación en los cargos más importantes. Al fundador le sucedió el profesor de instrucción pública Bartolomé Bohórquez Gil, pero causaría baja un año después por su traslado como docente a la ciudad de Tarifa. Allí este ubriqueño iba a continuar también con su actividad masónica tras afiliarse a la logia local Bercelius no. 199. Aunque no se rompían del todo los lazos con su primitiva logia puesto que iba a continuar figurando en su cuadro como miembro de honor y Garante de Amistad del taller tarifeño. Además en esta ciudad iba a desarrollar una importante labor social, cultural e incluso política dentro de las filas del

\footnotetext{
${ }^{4}$ Eduardo Enríquez del Árbol, "Problemática que plantea la multiplicidad de logias en Andalucía Occidental (provincia de Cádiz)", en La Masonería en la Historia de España (Zaragoza: Diputación General de Aragón, 1985), 225-238; Enríquez del Árbol, "La Masonería en la provincia de Cádiz en el siglo XIX: las logias de Grazalema, Villamartín y Ubrique", Revista Papeles de Historia 3 (1994): 37-51. María Pinto Molina, La masonería en Málaga y provincia (último tercio del siglo XIX) (Granada: Universidad, 1987), 136-158; y Morales Benítez, "La masonería en el medio rural. Implantación e incidencia social en la Sierra de Cádiz durante la coyuntura finisecular", en La masonería española y la crisis colonial del 98, coord. José Antonio Ferrer Benimeli (Zaragoza: CEHME, 1999), Tomo II, 845-863.

${ }^{5}$ Morales Benítez y Fernando Sigler Silvera, "Aportación al estudio de la Masonería en el medio rural andaluz: la logia América $\mathrm{n}^{\mathrm{o}} 27$ de Ubrique (Cádiz )", en Masonería, Política y Sociedad, coord. Ferrer Benimeli (Zaragoza: CEHME -Junta de Andalucía, 1989); Tomo II, 1101-1109.

${ }^{6}$ Se ha apuntado el posible parentesco con el general rebelde Ramón Leocadio Bonachea, natural de Remedios, fusilado por los españoles en La Habana en 1885. Sobre este personaje vid. Manuel de Paz, "Bandolerismo social e intentonas revolucionarias (1881-1893): la otra guerra de Cuba", en Revista Tebeto II, Anuario del Archivo Histórico Insular de Fuerteventura (1989).

${ }^{7}$ Archivo Histórico Nacional, Madrid, sección Ultramar, leg. 3.622. Sobre este oriente: Juan Manuel Castellano Gil, La masonería española en Cuba (Santa Cruz de Tenerife: Centro de la Cultura Popular Canaria, 1996) y Morales Benítez y Sígler Silvera, "La masonería gaditana de obediencia cubana: La logia Tolerancia y Fraternidad", en Masonería española y América, coord. Ferrer Benimeli (Zaragoza: CEHME, 1993), Tomo I, 313-322.
} 
partido liberal. Tras su etapa tarifeña, se instalará en Valencia para convertirse en una de las figuras más relevantes del teosofismo español y del librepensamiento ${ }^{8}$.

En tanto que durante estos años la logia de Ubrique iba a destacar como foco educativo y cultural que cristalizaría con la creación de una escuela laica, denominada Ateneo, que impartía todas las materias a alumnos con pocos recursos, así como con otra entidad dirigida a adultos. Esta línea de actuación se completó con la creación de una biblioteca y de un círculo cultural para atender también a personas adultas. Todo ello con un carácter abierto y gratuito tendente, ante la falta de otras alternativas, a proporcionar educación a los menos favorecidos ${ }^{9}$. Según el masón Arenas Vinagre la falta de ilustración estaría en el origen de los males de la sociedad y para remediar estos males: "La masonería puede y deber establecer centros de instrucción para, por este medio, sacar al trabajador de la ignominia y hacer de él un hombre instruido y libre" ${ }^{\prime \prime}$.

Con este fin se crearía un primer núcleo, el Círculo Instructivo Luz de la Sierra, fundado el 28 de diciembre de 1888. Ese día su Junta General eligió como primer presidente a Cristóbal Gutiérrez Bohórquez, simb. Bohórquez Gómez, y secretario a Manuel Janeiro Córdoba, simb. Huebaldo. El 3 de febrero de 1889 se daba cuenta de ello al Gran Maestre del Grande Oriente Español (GOE), Miguel Morayta, así como de su elección como presidente honorario de la nueva entidad.

A partir de este organismo, y dependiendo de él, se creará una escuela laica y de cuyas primeras gestiones tenemos noticias a partir del mes de mayo ${ }^{11}$. Finalmente, esta escuela para adultos, denominada Regeneración, inició su primer curso académico el 21 de octubre de 1889 con una matrícula de 45 alumnos de entre 10 y 40 años. Este número se irá incrementando progresivamente durante los meses posteriores hasta alcanzar dos años después 95 matriculaciones. Al mismo tiempo permanecía abierta la escuela Ateneo, que impartía disciplinas más especializadas, como nociones de geografía, de psicología y de zoología, así como una asignatura denominada Causas del Indeferentismo Religioso ${ }^{12}$. Además en los propios locales del círculo se inició la formación de una biblioteca abierta a toda la población y que se iba a ir incrementando con las aportaciones de los socios y nuevas adquisiciones ${ }^{13}$.

\footnotetext{
${ }^{8}$ Morales Benítez, "Bartolomé Bohórquez Gil. Teósofo y masón”, Aljaranda 50 (2003): 24-30.

${ }^{9}$ Morales Benítez y Sígler Silvera, "Aportación al estudio de la Masonería en el medio rural andaluz", 11011109 .

${ }^{10}$ Centro Documental de la Memoria Histórica (CDMH), Salamanca, Masonería, leg. 744-A-1

${ }^{11} \mathrm{CDMH}$, Salamanca, Masonería, leg. 744-A-1, carta de 22/5/1889. Las dignidades de América justificaban su tardanza en contestar al Gran Consejo por las ocupaciones que tenía en el Círculo debido a la creación de una escuela laica. La cuestión que les preocupaba era la constitución de una junta en la que debían participar también personas ajenas a la masonería.

${ }^{12}$ CDMH, Salamanca, Masonería, leg. 744-A-1, carta de 31/ 10/ 1889de la logia al Gran Consejo de la Orden.

${ }^{13}$ Sabemos que se adquirió, por ejemplo, la Historia de España del propio Miguel Morayta, catedrático de historia y gran maestre del GOE.
} 
A fines de ese mismo año la logia expresaba su satisfacción por la buena acogida que habían tenido todas estas iniciativas entre los miembros de los órganos rectores de la obediencia $^{14}$, quienes a su vez habían acordado transmitir a los masones ubriqueños su reconocimiento por los trabajos desarrollados. Este hecho, así como el rápido crecimiento en el número de alumnos en la escuela de adultos, iba a permitir a los miembros de América presumir de haber provocado el declive de otro centro educativo dependiente de la Iglesia y de un círculo obrero católico que había tenido que cerrar. En la carta enviada al Gran Consejo se dejaba ver la implicación de los masones con esta obra tan necesaria para la regeneración de la sociedad:

“(...) teniendo que luchar con un pueblo en que todavía están muy arraigadas ciertas preocupaciones que no dejan de producirnos los disgustos subsiguientes, que en su alto criterio pueden apreciar, alagar en alto grado nuestro amor propio no solo el aprecio que hacéis de ello, sino el resultado que en bien de todos vamos consiguiendo quizás más que era de esperar; pues a medida que nuestra escuela se va desarrollando, ha ido creciendo otra que había fundado el clericalismo hasta el punto de tenerla hoy cerrada, quizás para no abrirla más, así como un círculo que se titulaba de obreros católicos con todo y tener consignado por el Ayuntamiento 200 pesetas (consignación que es muy posible pase a nosotros con lo que daríamos otro desengaño más)"15.

Todas estas iniciativas, enmarcadas dentro de una estrategia de intervención en la vida pública través de estas instituciones culturales, entre las cuales el verdadero motor era el círculo, considerado como baluarte de la libertad en esta villa y faro luminoso, respondería a los deseos de extender sus ideales al resto de una población necesitada de esta regeneración moral. En este sentido la logia manifestaba que su objetivo no era otro que remover los obstáculos que impedían el desarrollo de esta población a través de la creación de una nueva generación de ciudadanos que se pudiesen formar en estas instituciones patrocinadas por la logia y capaces de operar el cambio que necesitaba la sociedad para orientarla por el camino del progreso: “(...) aumentar el círculo más y más para que de él y de la logia salgan los hombres que moralicen las conciencias y por lo tanto pacifiquen la atmósfera que rodea a esta villa (...) que paralizan su marcha progresiva".

Una prueba de esa vocación de actuar más allá de las columnas del templo masónico podríamos encontrarla en la propia configuración de todo este entramado organizativo por la interrelación que se observa entre las instituciones masónicas y profanas patrocinadas por el taller. Círculo instructivo, escuela, biblioteca y templo compartían el

\footnotetext{
${ }^{14}$ CDMH, Salamanca, Masonería, leg. 744-A-1, carta de 2/ 12/ 1889. La logia se congratula del acuerdo del Gran Consejo que reconocía estos trabajos y que le sería comunicada en plancha del 16/ 11/ 1889.

${ }^{15} \mathrm{CDMH}$, Salamanca, Masonería, leg. 744-A-1.
} 
mismo edificio. Las tres primeras instituciones se ubicaban en la planta baja, en tanto que en la de arriba estaba la sede de la logia.

En abril de 1891 en su memoria anual, el taller ubriqueño hacía un balance de los primeros tres años de actividad del círculo instructivo, que "sin carácter político, representa nuestros ideales", así como de las demás instituciones culturales sostenidas por América. Se llamaba la atención del nivel académico alcanzado, la buena acogida que seguía teniendo entre la población, así como el hecho de que "hace sentir su influencia no solo en esta población sino en las limítrofes" $"$.

Pero no podían ocultarse los problemas financieros y el enorme esfuerzo que exigía a los miembros de la logia el mantenimiento de todo este conjunto de instituciones educativas y culturales. Unos costos que recaerían en primer lugar sobre los bolsillos de los propios masones. El ayuntamiento contribuía con una subvención consistente en 25 pesetas mensuales pero, según los miembros de América, "llega tarde, mal y nunca" y cubría sólo una parte de los gastos que generaba la escuela laica, que impartía sus clases de forma gratuita. Durante el curso de 1891 la asistencia no bajaba de 90 alumnos y se hizo necesario nombrar a un ayudante para el profesor de primera enseñanza encargado de atenderlos. Ello incrementaba los gastos de forma considerable porque serían ahora dos los docentes a retribuir.

Los problemas de financiación se intentaron solucionar con la creación de una sociedad cooperativa y de ahorros impulsada por la propia logia. Esta entidad tenía que proporcionar los recursos necesarios mediante la capitalización en acciones de los propios fondos de la logia que debían constituir la base de esta sociedad. A partir de ahí los restantes socios, masones o no, debían suscribir a título individual las acciones a 25 pesetas cada una, pudiéndose subdividir éstas en medias o cuartos de acciones. El objeto era el sostenimiento del círculo, pero dependiendo de la marcha de la nueva entidad financiera impulsada por América, cuya constitución se anunciaba para primeros de mayo de 1891, se pretendía también ofertar artículos de primera necesidad al pueblo de Ubrique con el menor interés posible. De paso se cumpliría también otro de los objetivos perseguidos por los masones locales, como es "estrechar los lazos con el mundo profano". Sin embargo estos propósitos no llegarían a consolidarse puesto que la sociedad iba a tener una vida breve y anémica, según la propia logia, debido a las numerosas contrariedades ocasionadas por "la falta de comprensión, la apatía y la indolencia con que se miran en el mundo profano todas estas innovaciones y el poco aprecio que se siente por los sacrificios"17.

\footnotetext{
${ }^{16} \mathrm{CDMH}$, Salamanca, Masonería, leg. 744-A-1, Memoria de los trabajos de América correspondiente al período 1890-1891, 27/ 4/ 1891 .

${ }^{17}$ CDMH, Salamanca, Masonería, leg. 744-A-1, Memoria de los trabajos realizados por la Muy Leal Benem. y Resp. Log. América $n^{\circ} 27$ durante el año de 1891 a 1892, carta de 20/4/1892. Un año después de su creación se informaba que la Sociedad fue disuelta "para plazo más o menos largo".
} 
Pese a todo, parece que no decayó el entusiasmo de los masones que acordaban que el círculo Luz de la Sierra se hiciese cargo del sostenimiento de la escuela mediante la organización de rifas o la aportación individual de los socios, cuyo número se había incrementado debido al prestigio que estaba alcanzado la institución educativa y la influencia conseguida puesto que "va ya pesando alguna cosa en la localidad". Por lo que la logia, a pesar de los agobios económicos, podía enorgullecerse de proclamar que el ideal masónico de fraternidad era una realidad en el interior de una institución en la que coincidían representantes de diversos sectores sociales: “(...) todas las clases de la sociedad, desde el modesto obrero, la industria y la mejor acomodada, los cuales todos unidos ayudan al sostenimiento de la escuela de adultos donde cada día se destaca más la claridad que esparce la enseñanza y la fe y el amor que cada vez más inspira a todos nuestros conciudadanos" $"$.

Otro de los campos de actuación será la biblioteca del círculo, considerada la más rica de la localidad, "no solamente por el número de sus obras sino por la importancia de muchas de estas" y que se incrementaba con nuevos volúmenes. Así, en la memoria de 1892 se hacía constar las gestiones de un miembro de la logia para adquirir una biblioteca que se vendía en la ciudad de Cádiz y cuyos 120 volúmenes pasarían finalmente a engrosar los fondos del círculo ubriqueño.

Pero estos éxitos provocaron la desconfianza de algunos sectores de la localidad, así como de otras sociedades de signo opuesto, que no perdían ocasión para criticarlos, o bien tensiones con el clero local a causa de la enseñanza laica que se impartía en la escuela. Ante ello, la logia quería cerrar filas para "combatir a los que quieren imponer por toda instrucción una religión gastada por muchos retoques, tiene la masonería que fomentar a toda costa la enseñanza laica, base de todo progreso".

Asimismo, las autoridades civiles dejaron de conceder la subvención con la que contribuían al sostenimiento de la escuela. La logia decía que la respuesta que debía darse a esta política oscurantista de "unos en política coartando la libertad de acción y (de) los otros en el púlpito y donde encuentran ocasión (pero siempre en la sombra) nos sahieren y anatematizan" no podía ser otra que continuar combatiendo desde el interior de sus columnas, a falta de otras fuerzas, la intolerancia religiosa o política. Como prueba de los frutos de esta lucha estarían los numerosos actos civiles organizados por los miembros de América o la continuidad de la escuela pese a las dificultades de todo tipo a las que tenía que hacer frente ${ }^{19}$. Incluso se marcaba como objetivo aumentar las actividades del Círculo Instructivo para "que de él y de la logia salgan los hombres que moralicen las conciencias y por lo tanto purifiquen la atmósfera que rodea a estos Valles".

\footnotetext{
${ }^{18} \mathrm{CDMH}$, Salamanca, Masonería, leg. 744-A-1.

${ }^{19}$ CDMH, Salamanca, Masonería, leg. 744-A-1, Memoria de 17/4/1893. Se resta importancia a la retirada de la ayuda "sin que para nada nos hagan falta los pocos metales que por espacio de algunos meses nos venía dando el municipio para el sostenimiento de la misma".
} 
Finalmente, y como consecuencia de la retirada de la subvención municipal, el quinto año de vida de esta escuela pudo comenzar sólo gracias a una suscripción que encabezaron los propios miembros del taller con una aportación personal consistente en 150 pesetas. La logia había celebrado previamente una tenida extraordinaria para dar respuesta a la nueva situación. Se decía que en esta tarea no estaban solos, sino que le secundaban "muchos amantes del progreso (...)" que también contribuían económicamente y que les llenaba de satisfacción, viéndose "colmadas por este año nuestras aspiraciones". La apertura del curso no se efectuaba el día primero de octubre, como otros años, sino que de acuerdo con la junta de instrucción se retrasaba unos días para hacerla coincidir con otra celebración con motivo del IV centenario del descubrimiento de América. Y así la noche del 23 de octubre de 1892, además del citado acto, se celebró una velada en homenaje a Colón, que reunió en los locales del Círculo a numerosas personas entre invitados y alumnos. La logia quería participar de esta forma en las celebraciones que había impulsado el GOE en Madrid con este mismo motivo ${ }^{20}$, el denominado festival masónico en conmemoración del descubrimiento de América, mediante una gran tenida blanca en un salón de la capital Madrid con una gran solemnidad y asistencia de público.

En Ubrique, la logia América quiso emular esta iniciativa con la celebración de un acto destinado a alcanzar gran relevancia en la localidad, sin duda poco acostumbrada a eventos de este tipo. Actuaron de anfitriones la directiva y los socios del círculo instructivo, quienes recibieron a las autoridades, comisiones del círculo liberal, de artesanos y numerosos invitados. El presidente de la junta de instrucción Antonio, P. Hinojosa y Ruiz, sería el primero en hacer uso de la palabra y posteriormente otros oradores también aprovecharon la ocasión para incidir en las ideas de la relación que existe entre la instrucción y el progreso de los pueblos y los beneficios que proporcionan las "leyes progresivas", para terminar haciendo votos a fin de mantener una institución de tanta importancia para muchas personas de la localidad que de otra manera estarían "condenados a vivir en la ignorancia".

La crónica de este acto sería recogido en las páginas del boletín oficial del GOE que reproduce en su integridad la carta enviada por los masones de Ubrique. Un extenso artículo en el que se destaca su relevancia y que puede darnos también una idea del talante abierto con el que querían actuar los masones ubriqueños:

"En esa noche apareció la extensa fachada del Círculo iluminada con tal profusión que el público se agolpaba a contemplar tan soberbio espectáculo; los tres balcones de nuestro Templo, que ocupa la planta alta del Círculo, estaban adornados con

\footnotetext{
${ }^{20}$ La logia no tenía recursos para enviar representación a Madrid, por lo que no figura oficialmente entre las logias representadas, pero la casualidad de un viaje por motivos familiares de un miembro de América quiso que finalmente fuese testigo de dichos actos a nivel nacional. Se trataba del veterinario Gabriel Martín Martínez, simb. M. Servet, quien posteriormente llevaría esta idea a Ubrique.
} 
tanto gusto y esmero, que entre los simbólicos ramos de acacia, había caprichosas luces formando compases, escuadras, triángulos, etc., cuyos atributos contemplaba absorta la muchedumbre, despertando en ella la consiguiente curiosidad. El salón de recreo no estaba menos engalanado, destacándose en el centro, y entre ricas colgaduras, espejos y rodeados de acacia, la venerable figura de Colón. En el pequeño jardín que poseemos, que da acceso al salón destinado a la Escuela, lucieron gran profusión de luces, y por último, el espacios o salón de la Escuela con sus sencillos adornos de mapas, pizarras, carteles y otros objetos destinados a la enseñanza, y en el centro un hermoso cuadro que representa la Masonería alumbrando el mundo. A las siete de la noche los acordes de la música que ocupaba el jardín anunciaban que se iba a proceder a la apertura de la susodicha escuela $(\ldots)^{21,}$.

Sin embargo, este curso, que comenzaba de una forma tan festiva, no estaría exento de dificultades y sólo pudo completar su período lectivo gracias a un nuevo esfuerzo económico de los miembros de América que tuvieron que financiarlo en su integridad puesto que la suscripción abierta no había dado los resultados esperados. La logia no dudaba en achacarlo a las penurias económicas por "la crisis calamitosa que todas las clases venimos sufriendo".

Todas estas incidencias irían mermando cada vez más las arcas de la logia, que no sin grandes esfuerzos podía mantener todas las instituciones culturales y educativas que patrocinaba. Y difícilmente podría cumplir dentro de los plazos previstos con sus obligaciones tributarias con el Grande Oriente de España, por lo que se tenía que solicitar a la obediencia diversas moratorias, así como justificar sus reiterados retrasos en el pago de las cuotas masónicas. Inmersos en esta coyuntura crítica, a partir de junio de 1895 el taller iniciaba un periodo de inactividad que se prolongaría hasta 1901 y que habría que enmarcar dentro de la crisis general de la masonería española de finales de siglo. Ello iba a implicar la desintegración de toda su estructura organizativa, así como de las instituciones dependientes de ella.

Por otra parte, y en lo que se refiere a sus miembros más significativos durante estos años, conocemos la relación o cuadro lógico de América con los cargos que ostentaron dentro de la logia durante el $1893^{22}$.

\footnotetext{
${ }^{21}$ Boletín Oficial del Gran Oriente Español (BOGOE), noviembre de 1892, pp. 192-193. Se decía también que entre los invitados al acto estaba el profesor de instrucción primaria Francisco Fatou, quien se ofreció a los promotores de este centro para impartir un día a la semana la asignatura de historia de España. También se ofreció el médico y miembro de la logia Ramón de la Torre, simb. Ferrán, gr. $18^{\circ}$ a impartir geografía.

${ }^{22} \mathrm{CDMH}$, Salamanca, Masonería, leg. 744-A-1. Venerable maestro: Juan López Parra, simb. Galileo; primer vigilante: Félix Sánchez López, simb. Newton; segundo vigilante: José Domínguez Hurtado, simb. García Vao; secretario: Cristóbal Gutiérrez Bohórquez, simb. Bohórquez Gómez; secr. adj.: Miguel León Rodríguez, simb. H. Cortés; orador: Bartolomé Moreno Mena, simb. Garibaldi; orador adj.: Gabriel Martín Martínez, simb. M. Servet; tesorero: Francisco Zaldívar Gómez, simb. F. Garrido; limosnero: Estanislao Pérez Mancilla, simb. Victoria; maestro de ceremonias: José Gutiérrez Bohórquez, simb. Sixto Cámara; primer experto:
} 
En cuanto al impacto que tuvo la masonería en la comarca de la Sierra, más que un colectivo de exclusiva actividad política de tipo conspirador, como frecuentemente se le ha atribuido desde una determinada historiografía, se erigió en una sociedad integrada por miembros de clase media ilustrada con fines de regeneración social en función de principios de filosofía racionalista y de fraternidad universal y con un gran componente de promoción cultural.

Dentro de estos parámetros podemos encontrar diversos ejemplos de lo que representó el masonismo en la Sierra de Cádiz a finales del siglo XIX en base a concepciones o planteamientos distintos, puesto que iban a interpretarse también de diferente manera unas obligaciones masónicas que luego proyectarían a la sociedad. En este sentido América entendía que precisamente en la falta de ilustración del pueblo estaría el origen de todos los males que afectaban a la sociedad. Y así uno de sus miembros defendía en uno de sus escritos que para remediar en parte las desgracias de las clases pobres "la masonería puede y debe establecer centros de instrucción para, por este medio, sacar al trabajador de la ignorancia y hacer de él un hombre instruido y libre"23. En este contexto, la logia de Ubrique destacó por el papel que jugó como foco cultural y educativo en la población que se plasmaría en la creación de escuelas laicas, así como una biblioteca y un círculo cultural. Todas estas instituciones tuvieron un carácter abierto y gratuito, con ello la masonería ubriqueña quiso contribuir a proporcionar ilustración a las capas más desfavorecidas de la localidad sin acceso a la cultura.

\section{Conclusión}

En líneas generales se podría confirmar la interpretación de la masonería en este contexto de la serranía gaditana como ese instrumento utilizado por una clase media o pequeña burguesía ilustrada como vía de participación en su entorno político, social, cultural e ideológico intermedia de carácter democrático y progresista. Para la resolución de la denominada cuestión social, sin duda el asunto que más preocupa a los masones de América, la masonería ubriqueña plantea una profunda regeneración, una purificación, de la vida pública española que ha de ser dignificada. Por ello sitúan los fines de la institución masónica como "obra regeneradora y humanitaria (necesaria) para oponer un dique a tanta inmoralidad política, administrativa y religiosa" ${ }^{24}$.

Manuel Arenas Vinagre, simb. Proudhon; segundo experto: Federico Coveñas Orellana, simb. Numancia; guarda templo: Sebastián Villalobos Viruez, simb. Figueras.

${ }_{23}$ Morales Benítez, "Manuel Arenas Vinagre Manuel Arenas Vinagre, líder republicano y masón de Ubrique", Papeles de Historia 2 (1990): 139-146; y Morales Benítez, "Manuel Arenas Vinagre: un reformador social en el tránsito del siglo XIX al XX”, Ubrique Información 11, no. 8 (2003).

${ }^{24}$ CDMH, Salamanca, Masonería, leg. 744-A-1. 
En este contexto crítico, será necesario fomentar la ilustración. La cultura, en sentido positivo, debía ser capaz de corregir la sociedad porque "la ignorancia eterniza la esclavitud, la opresión y la tiranía". Por ello el fomento de actividades de difusión cultural y promoción educativa iba a ocupar un lugar importante en el quehacer de los masones de América. Y en este contexto, se emprende una labor de formación ideológica y cultural del proletariado.

\section{Fuentes}

Centro Documental de la Memoria Histórica, Salamanca, Masonería, leg. 744-A-1.

\section{Bibliografía}

Álvarez Lázaro, Pedro. La Masonería, escuela de formación del ciudadano. La educación interna de los masones españoles en el último tercio del siglo XIX. Madrid: Publicaciones de la Universidad Pontificia Comillas, 1996.

Álvarez Rey, Leandro. "Un espacio de sociabilidad: la masonería en Cádiz entre el 98 y la Guerra Civil”. En En torno al 98. Editado por en Rafael Sánchez Mantero. Huelva: Universidad de Huelva, 2000.

Castellano Gil, Juan Manuel. La masonería española en Cuba. Santa Cruz de Tenerife: Centro de la Cultura Popular Canaria, 1996.

Cruz Orozco, José Ignacio. Masonería y educación en la II República española. Alicante: Instituto de Cultura Juan Gil Albert, 1993.

Enríquez del Árbol, Eduardo. "Problemática que plantea la multiplicidad de logias en Andalucía Occidental (provincia de Cádiz)”. En La Masonería en la Historia de España. Zaragoza: Diputación General de Aragón, 1985.

Enríquez del Árbol, Eduardo. "La Masonería en la provincia de Cádiz en el siglo XIX: las logias de Grazalema, Villamartín y Ubrique”. En Revista Papeles de Historia 3 (1994): 37-51.

Morales Benítez, Antonio. "Manuel Arenas Vinagre, líder republicano y masón de Ubrique”. En Revista Papeles de Historia 2 (1990): 139-146.

Morales Benítez, Antonio. "La masonería en el medio rural. Implantación e incidencia social en la Sierra de Cádiz durante la coyuntura finisecular”. En La masonería española y la crisis colonial del 98. Coordinado por José Antonio Ferrer Benimeli. Zaragoza: CEHME, 1999.

Morales Benítez, Antonio. "Masonería y Educación. La propuesta pedagógica de la logia América de Ubrique a finales del siglo XIX”. En Revista Hespérides 15 (2013): 2226. 
Morales Benítez, Antonio y Fernando Sígler Silvera. "Aportación al estudio de la Masonería en el medio rural andaluz: la logia América no 27 de Ubrique (Cádiz )”. En Masonería, Política y Sociedad. Coordinado por José Antonio Ferrer Benimeli. Zaragoza: CEHME-Junta de Andalucía, 1989.

Morales Benítez, Antonio y Fernando Sígler Silvera. "La masonería gaditana de obediencia cubana: La logia Tolerancia y Fraternidad”. En Masonería española y América. Coordinado por José Antonio Ferrer Benimeli. Zaragoza: CEHME, 1993.

Paz, Manuel de. "Bandolerismo social e intentonas revolucionarias (1881-1893): la otra guerra de Cuba”. En Revista Tebeto II, Anuario del Archivo Histórico Insular de Fuerteventura (1989).

Pinto Molina, María. La masonería en Málaga y provincia (último tercio del siglo XIX). Granada: Universidad, 1987. 